\title{
The Impact of External Events on Trends in Breast Imaging
}

\author{
Kathleen M Capaccione ${ }^{1 *}$; Sophia Huang ${ }^{1}$; Elizabeth West ${ }^{1}$; Aileen Deng $^{2}$; Mary M Salvatore ${ }^{1}$; Elise Desperito ${ }^{1}$ \\ ${ }^{1}$ Department of Radiology, Columbia University Irving Medical Center, New York, NY, USA. \\ ${ }^{2}$ Department of Hematology/Oncology, Novant Health Cancer Institute, Novant Health, Mooresville, North Carolina, USA.
}

\author{
*Corresponding Author(s): Kathleen M Capaccione \\ Department of Radiology, Columbia University Irving \\ Medical Center, 622 W 168th Street, New York, New \\ York 10032, USA. \\ Tel: 908-672-6160; \\ Email:kmc9020@nyp.org
}

Received: Dec 20, 2021

Accepted: Jan 19, 2022

Published Online: Jan 24, 2022

Journal: Journal of Radiology and Medical Imaging

Publisher: MedDocs Publishers LLC

Online edition: http://meddocsonline.org/

Copyright: (C) Capaccione KM (2022). This Article is distributed under the terms of Creative Commons Attribution 4.0 International License

Keywords: Mammography; Breast Imaging; COVID-19; Screening.

\section{Abstract}

Objective: Here we analyze trends in breast cancer imaging over the past decade to evaluate the impact of changes in access to care and the COVID-19 pandemic on breast imaging utilization.

Methods: We queried our radiology record system to identify numbers of specific breast imaging studies from $01 / 01 / 2010$ to $03 / 31 / 21$. We retrospectively assessed yearly numbers of exams since 2010 and weekly numbers during the COVID-19 pandemic in the greater New York City region. We performed Wilcoxon signed ranks tests to compare three-month periods during the pandemic to the same time period the prior year.

Results: Ten-year trends in breast imaging showed an increase in screening mammography in the early part of the decade, which declined later in the decade. These changes corresponded to implementation of the Affordable Care Act in 2010 and the American Cancer Society Screening guidelines change in 2015. Breast MRI increased from 2017-2019, likely a delayed effect of research demonstrating a mortality benefit to high-risk women. Both screening mammography and breast MRI sharply declined during the height of the COVID-19 pandemic. Breast MRI has since rebounded, while screening mammography remains significantly reduced compared to 2019 exam numbers.

Conclusion: Breast imaging exam numbers are affected by external events. Most recently, the COVID-19 pandemic significantly decreased screening mammograms and these have not rebounded to pre-pandemic levels. Given the proven benefit of screening mammography, this could have serious long-term effects for patients and should be carefully followed to ensure that all women get needed breast imaging and care.

Cite this article: Capaccione KM, Huang S, West E, Deng A, Salvatore MM, et al. The Impact of External Events on Trends in Breast Imaging. J Radiol Med Imaging. 2022; 5(1): 1065. 


\section{Introduction}

Historically, there has been controversy regarding the use of screening mammography, most notably based on results of the Canadian National Breast Screening Study, a large randomized controlled trial of screening mammography which reported no benefit to women from 40-49 [1], and later reported a similar lack of benefit in women 50-59 years of age [2]. However, multiple studies demonstrated clear benefit of screening mammography [3-6]. A recent meta-analysis assessing reduced mortality as a result of screening mammography evaluated 58 primary sources and concluded that there was a definite reduction in mortality as a result of breast cancer screening [7].

There has also been controversy regarding the initial age at which to begin breast cancer screening and screening frequency since the inception of screening mammography $[3,8-$ 10]. A variety of institutions have weighed in with breast cancer screening guidelines during this time: American Cancer Society (ACS), American College of Obstetricians and Gynecologists (ACOG), American College of Physicians (ACP), American College of Radiology (ACR), American Medical Association (AMA), National Cancer Institute ( $\mathrm{NCl}$ ), National Comprehensive Cancer Network (NCCN), and the United States Preventative Task Force (USPTF).

ACS guidelines alone have changed several times since 1980. Before 1980, yearly mammography was recommended for women after 50 years of age. From 1980-1992, a baseline mammogram was recommended between 35-39 and screening frequency was at the discretion of the provider. From 19821997, mammography was recommended every $1-2$ years from ages $41-49$, then yearly after 50 . From 1997-2009, a screening mammogram was recommended yearly after 40 years of age [11]. In 2015, the American Cancer Society (ACS) updated their guidelines for breast cancer in women with average risk, recommending that a woman discuss with her primary care physician (PCP) the need for screening mammography between the ages of 40-44, initiation of annual screening mammography at 45 years of age, then transition to biannual screening at 54 years of age [12]. To date, little data is available regarding the effect this change had on utilization of screening mammography.

Not only has the debate over the efficacy of breast cancer screening in general and the debate over when and how often breast cancer screening should occur influenced breast cancer screening in the US, but other circumstances have also influenced breast cancer care in the US. In 2010, the Affordable Care Act (ACA) was signed allowing many patients who previously did not have access to medical care to obtain insurance. Subsequent research has shown that this resulted in more patients with a usual place of care and fewer who were unable to access care when needed [13]. An important aspect of the ACA with regard to screening mammography was the elimination of patient cost sharing in many health plans, colloquially known as "copay". A study by Trivedi et al., analyzed the effect of this change and found approximately a $5 \%$ increase in screening mammography in plans without patient cost-sharing, compared to essentially stable rates in those with cost-sharing [14]. These data provide a second potential cause of increases in breast imaging after 2010.

The COVID-19 pandemic has been an unprecedented event in modern healthcare, with millions infected worldwide $[15,16]$. Cancer care was significantly affected by the COVID-19 pandemic. In a study of more than 600 breast cancer patients in the United States, approximately $44 \%$ reported delays in care due to the pandemic [17]. A comprehensive study from England analyzed weekly cancer diagnostic referrals and chemotherapy treatment data for 24 cancer sites and estimated that there would be 6,270 excess deaths in England and 33,890 excess deaths in the United States at 1 year post analysis [18]. A study of United States data on six common types of cancer evaluated new cancer diagnoses before and during the pandemic and found significant declines in the diagnosis of all cancer types studied, with an overall decrease of $46.4 \%$. Breast cancer diagnosis was the most affected and fell 51.8\% [19]. Early population-based data demonstrated a decline in screening mammography in 2020 compared to prior years attributed to the pandemic [20].

A recent study published in the Journal of the National Cancer Institute by Sprague, et al., analyzed national data on screening and diagnostic mammograms from 62 radiology centers [21]. They found that utilization of these studies was significantly decreased in April of 2020, but began to rebound by July of 2020 . Here, we build upon their work with a more detailed look at a tertiary medical center and its affiliate hospitals' experience with breast imaging utilization throughout the past decade including the pandemic. We continue this analysis though March of 2021 to assess whether breast-imaging utilization had completely rebounded one year after the declaration of the pandemic. Finally, we discuss how events of the last decade might reflect breast cancer care over time.

\section{Methods}

\section{Data collection}

A Human Research Protection Office and Internal Review Board Letter of Exemption was obtained to study imaging trends over time; exemption was granted given that no individual patient data was accessed. Key specific breast imaging studies were identified and the radiology record system queried for studies with labels corresponding to these exams, which included screening mammograms and contrast enhanced breast MRI obtained for any indication. Data was drawn from a major tertiary medical center and two affiliate community hospitals. Data was recorded as yearly totals from $01 / 01 / 2010$ to $12 / 31 / 2021$ and weekly totals for one year beginning with the World Health Organization's declaration of the pandemic on March $11^{\text {th }}, 2020$ and retrospectively for one year prior.

\section{Patient selection}

M*Model Catalyst (Maplewood, MN, U.S.), a radiology record search engine, was used to query the prior radiology reports for exams with specific titles, as described above. Only female patients were included in this analysis. No other criteria were used to exclude patients from this study.

\section{Statistical analysis}

Statistical analysis was performed in conjunction with the Mailman School of Public Health at Columbia University. Statistical analysis was performed in Microsoft Excel v.16.48 (Redmond, WA, USA) and Stata 16.1 (College Station, TX, USA). Averages and Standard Deviations (SD) were calculated for each sample. For yearly numbers, data was normalized to 2010 exam numbers and expressed as a percent change. Weekly values were divided into quarters (subsequently denoted Q1-4) for analysis and 2020 values were compared to 2019 values using a Wilcoxon Signed Rank Test. P values $<.05$ were considered sta- 
tistically significant.

\section{Results}

\section{Ten-year trends in breast imaging utilization}

Our data demonstrated that breast-imaging trends were dynamic over the past decade. Figure 1 demonstrates trends in screening mammography (A) and breast MR (B). Screening mammography increased in the early part of the 2010's, after which there was a decline in the number of screening mammograms. This coincided with the implementation of the ACS's new screening guidelines detailed above. Numbers of screening mammograms declined in 2020, coinciding with the COVID-19 pandemic. Breast MRI utilization was essentially stable until 2017, at which time there was a marked increase in utilization. There was a sharp decline in screening mammography obtained in 2020 during the COVID-19 pandemic.

\section{Effect of COVID-19 pandemic on breast imaging utilization}

Given the marked effect of the COVID-19 pandemic on yearly number of breast imaging exams, weekly utilization of breast imaging exams was studied in 2020 during the pandemic compared to one year prior. Figure $2 \mathrm{~A}$ demonstrates weekly screening mammography numbers which exhibited a statistically significant decline during the height of the COVID-19 pandemic in the greater New York region (Wilcoxon Signed Rank Test, $p=.0002$ ). This decline rebounded in June of 2020, correlating with New York's entry into Phase I of recovery. However, by the end of the study period screening mammography rates still had not completely recovered to pre-pandemic levels, with statistically significantly fewer exams in each quarter of 2020 (Q2 $\mathrm{p}=.0051 ; \mathrm{Q} 3 \mathrm{p}=.0049 ; \mathrm{Q} 4 \mathrm{p}=.0002$ ).

Analysis of breast MRI exams demonstrated a similar statistically significantly decline in Q1 $(p=.0012)$. However, unlike screening mammography breast MRI exam numbers completely rebounded by Q2 of 2020 ( $p=.1870)$ and remained similar to 2019 levels for the remainder of the study period (Q3 $p=.3674$, Q4 $p=.5527)$.

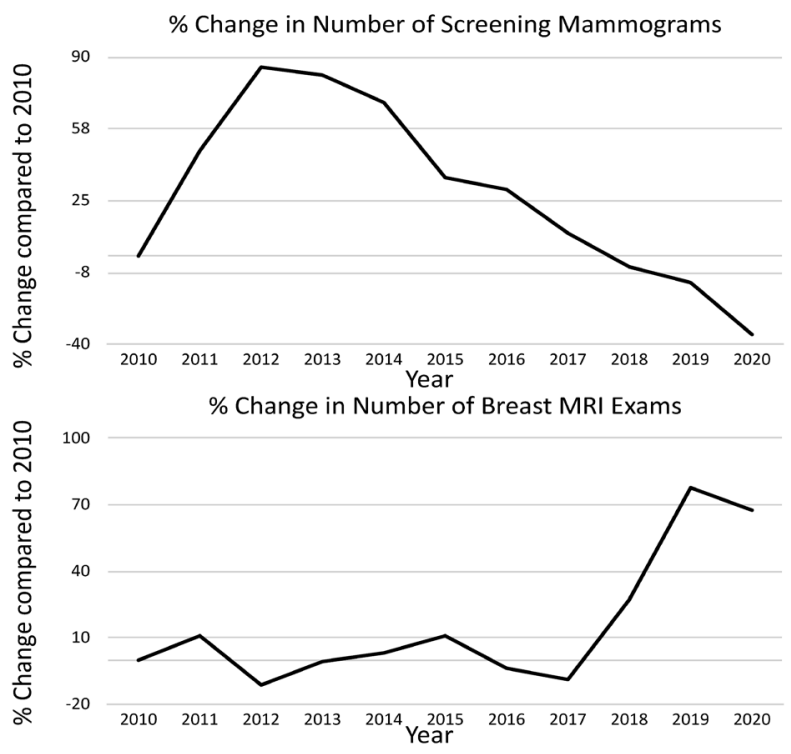

Figure 1: Trends in breast imaging studies over time expressed as percent change compared to 2010 exam numbers. Screening mammography increased in the early part of the 2010's, then began to decline. Breast MRI was steady in the early part of the decade, with increased utilization beginning in 2017. Both exam types decreased after declaration of the COVID-19 pandemic.
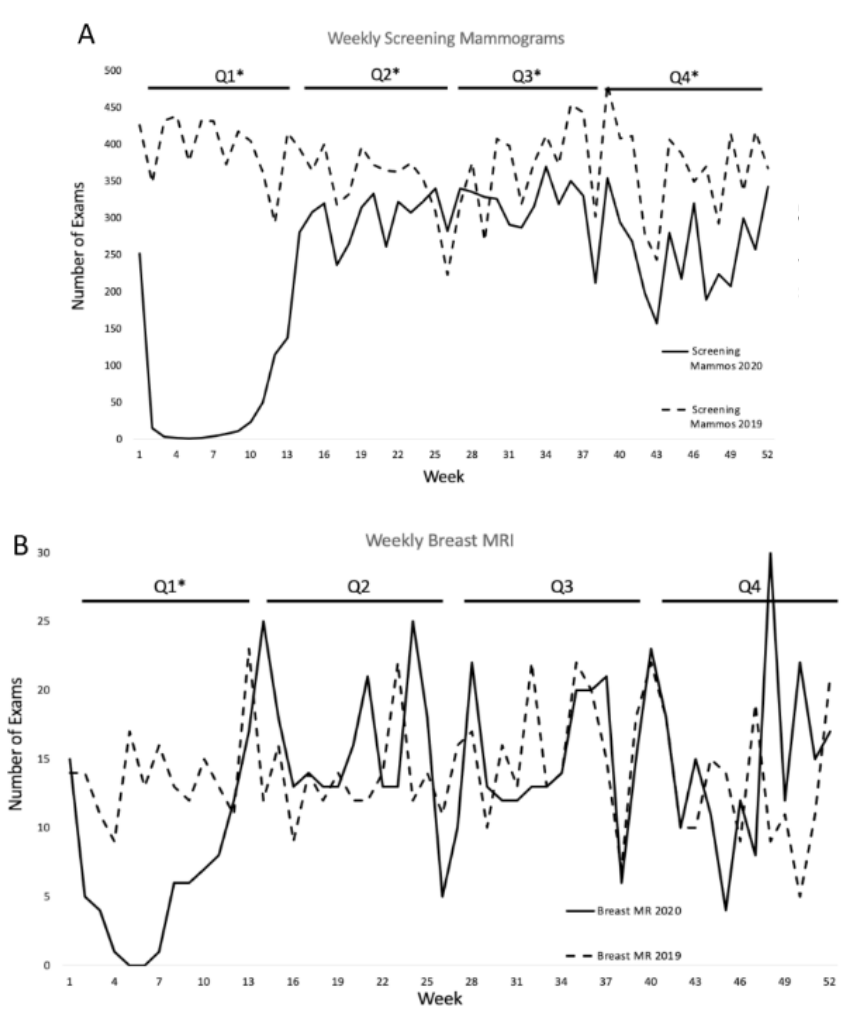

Figure 2: Weekly trends in breast imaging demonstrated marked decreases in numbers of exams for (A) screening mammograms and (B) breast MRI exams in Q1 of 2020 compared to 2019, beginning at March $11^{\text {th }}, 2019$ and 2020. All studies showed a rapid decline in 2020 utilization just after the pandemic was declared. While breast MRI rebounded to pre-pandemic levels in Q2-4, there were significantly fewer screening mammography exams in each quarter of the year after declaration of the pandemic. Quarters in 2020 that differed significantly compared to 2019 are marked*.

\section{Discussion}

Breast imaging plays a critical role in the detection and management of breast cancer. Screening mammography results in more cancers detected early and a lower incidence of late stage disease [22]. Breast imaging utilization has been dynamic over the past decade, changing in response to external events. Implementation of the Affordable Care Act in 2010 increased access to care for many patients both directly and indirectly by eliminating patient cost-sharing $[14,23]$. These changes likely account in part for the continued increase in screening mammography in our study cohort throughout the early part of the decade.

The 2015 American Cancer Society Screening Mammography guidelines [12] had a major impact on the trajectory of breast cancer screening utilization. Given the short time interval since implementation, limited data is presently available regarding the effects of implementation of the new 2015 ACS screening recommendations. Here, we demonstrate a decline in the number of screening mammograms obtained in the years after the implementation of these recommendations. Also contributing to the peak number of exams in 2015, according to the US Census bureau, New York City's population rose from 2010 to 2016, at which time it began to decline [24]. It is likely that the combination of both these factors contributed to the decline of screening mammography throughout the latter part of the decade in our region. 
These data demonstrate that breast MRI markedly increased in 2017. In 2017, the American Society of Breast Surgeons issued consensus guidelines on the use of Screening and Diagnostic MRI imaging of the breast [25]. We hypothesize that this may have raised awareness among breast surgeons for breast $\mathrm{MRI}$ indications and led to increased ordering at that time. Prior to this, in 2010, Carla Boetes published a comprehensive article reviewing evidence on the use of breast MRI, concluding that it has higher sensitivity for the detection of breast cancer in younger women with an increased risk of breast cancer [26]. We postulate that the increases toward the end of the decade were also influenced by this sentinel paper, and that the time between publication and increased number of exams reflects the time taken for clinicians to implement this new screening modality for breast imaging. More generally, improvements in the image quality and acquisition in breast MRI likely led to increased numbers of exams obtained.

Here, we demonstrate a marked decline in screening mammography and breast MRI during the COVID-19 pandemic. Data from New York City suggests that the peak hospitalizations for COVID-19 occurred during the first and second week of April, 2020 [27], coinciding with the lowest point of the curves for the imaging modalities described above. While not surprising, the shift from normal breast imaging patterns may have significant implications on the number of cancers diagnosed and treated [28]. Research in other fields has demonstrated marked decrease in access to breast cancer care as a result of the pandemic. A study from Italy demonstrated an $87 \%$ decrease in breast surgery during the first month of the COVID-19 pandemic $[29,30]$. Our data are in concert with these studies, providing the radiologic perspective on the overall diminution in available breast cancer care during the COVID-19 pandemic.

Importantly, our data demonstrated that screening mammography levels had not rebounded to pre-pandemic levels one year after declaration of the pandemic. In the early period of the pandemic, many patients did not seek care given fear of community transmission [31,32]. However, medical centers have made adjustments to practice settings resulting in patients returning for care in near normal numbers. Our data raises concern that the interruption in regular screening may have resulted in patients forgoing screening mammography altogether, which could result in missed early breast cancer and worse outcomes later on. Continued attention to these trends as well as active efforts to promote patients' return for screening will be an important part of breast imaging in the coming years.

Limitations of this study include a limited sample size taken from a small number of mammography centers in a single healthcare system. Data for this study was obtained in the greater New York City region, which was one of the regions most severely affected by the pandemic, limiting generalizability. As a retrospective review, this study provided a picture of historic imaging trends, however a prospective study would include additional information on trends in breast imaging beyond the $\mathrm{CO}$ VID-19 pandemic. Further, while this study looked at aggregate numbers of breast imaging studies, a more detailed prospective investigation of individual patients with deferred breast imaging would have shed light on whether the decline in imaging use represented delays in care or if patients were ultimately lost to follow-up. Despite these limitations, we believe that these data provide robust information regarding trends in breast imaging over the past decade and how external effects can play a significant role in these trends.

\section{Conclusion}

Both the changes in guidelines and access to screening mammography as well as the COVID-19 pandemic have had a significant impact on breast cancer imaging utilization. Screening mammography levels experienced an expected decline during the height of the pandemic, but importantly screening rates have not returned to their pre-pandemic levels, implying that some women are not receiving adequate preventative care. Changes in screening guidelines and access to care increased access to mammography in the early part of the 2010's, and it is important for the breast care community not to lose these gains due to disruption from the pandemic. Given the correlation with screening mammography and breast cancer incidence and mortality, these trends may have significant implications for women's health for years to come.

\section{Declarations}

Acknowledgements: This publication was supported by the National Center for Advancing Translational Sciences, National Institutes of Health, through Grant Number UL1TR001873. The content is solely the responsibility of the authors and does not necessarily represent the official views of the $\mathrm{NIH}$.

Conflict of interest: Mary M. Salvatore- Speaker and Consultant: Genentech, Boehringer Ingelheim. Grant funding: Genentech, Boehringer Ingelheim. The remaining authors have no conflicts to disclose.

\section{References}

1. Miller AB, Baines CJ, To T, Wall C. Canadian National Breast Screening Study: 1 . Breast cancer detection and death rates among women aged 40 to 49 years. Cmaj. 1992; 147: 14591476.

2. Miller $A B$, Wall $C$, Baines $C J$, Sun $P$, To $T$, et al. Twenty five year follow-up for breast cancer incidence and mortality of the Canadian National Breast Screening Study: randomised screening trial. Bmj. 2014; 348: g366.

3. Autier P, Boniol M, Gavin A, Vatten LJ. Breast cancer mortality in neighbouring European countries with different levels of screening but similar access to treatment: trend analysis of WHO mortality database. BMJ. 2011; 343: d4411.

4. Breast Cancer Screening: A Summary of the Evidence for the U.S. Preventive Services Task Force. Annals of Internal Medicine. 2002; 137: 347-360.

5. Olsen O, Gøtzsche PC. Cochrane review on screening for breast cancer with mammography. The Lancet. 2001; 358: 1340-1342.

6. Nyström L, Andersson I, Bjurstam N, Frisell J, Nordenskjöld B, et al. Long-term effects of mammography screening: updated overview of the Swedish randomised trials. The Lancet. 2002; 359: 909-919.

7. Mandrik O, Zielonke N, Meheus F, Severens JLH, Guha N, et al. Systematic reviews as a 'lens of evidence': Determinants of benefits and harms of breast cancer screening. International Journal of Cancer. 2019; 145: 994-1006.

8. Olsen $\mathrm{O}$, Gotzsche PC. Cochrane review on screening for breast cancer with mammography. Lancet. 2001; 358: 1340-1342.

9. Humphrey LL, Helfand M, Chan BK, Woolf SH. Breast cancer screening a summary of the evidence for the U.S. Preventive Services Task Force. Ann Intern Med. 2002; 137: 347-360.

10. Nystrom L, Andersson I, Bjurstam N, Frisell J, Nordenskjold B, et al. Long-term effects of mammography screening: updated 
overview of the Swedish randomised trials. Lancet. 2002; 359: 909-919.

11. Society AC. History of ACS Recommendations for the Early Detection of Cancer in People without Symptoms. American Cancer Society. 2020.

12. Oeffinger KC, Fontham ET, Etzioni R, Herzig A, Michaelson JS, et al. Breast Cancer Screening for Women at Average Risk: 2015 Guideline Update From the American Cancer Society. Jama. 2015; 314: 1599-1614.

13. Glied S, Ma S, Borja AA. Effect of the Affordable Care Act on Health Care Access. Issue Brief (Commonw Fund). 2017; 13: 1-11.

14. Trivedi AN, Leyva B, Lee Y, Panagiotou OA, Dahabreh IJ. Elimination of Cost Sharing for Screening Mammography in Medicare Advantage Plans. N Engl J Med. 2018; 378: 262-269.

15. Sohrabi C, Alsafi Z, O'Neill N, Khan M, Kerwan A, et al. World Health Organization declares global emergency: A review of the 2019 novel coronavirus (COVID-19). Int J Surg. 2020; 76: 71-76.

16. Lu H, Stratton CW, Tang Y-W. Outbreak of pneumonia of unknown etiology in Wuhan, China: The mystery and the miracle. Journal of Medical Virology. 2020; 92: 401-402.

17. Papautsky EL, Hamlish T. Patient-reported treatment delays in breast cancer care during the COVID-19 pandemic. Breast Cancer Research and Treatment. 2020; 184: 249-254.

18. Lai AG, Pasea L, Banerjee A, Denaxas S, Katsoulis M, et al. Estimating excess mortality in people with cancer and multimorbidity in the COVID-19 emergency. 2020.

19. Kaufman HW, Chen Z, Niles J, Fesko Y. Changes in the Number of US Patients With Newly Identified Cancer Before and During the Coronavirus Disease 2019 (COVID-19) Pandemic. JAMA Network Open. 2020; 3: e2017267-e2017267.

20. Peng S-M, Yang K-C, Chan WP, Wang YW, Lin LJ, et al. Impact of the COVID-19 pandemic on a population-based breast cancerscreening program. Cancer. 2020; 126: 5202-5205.

21. Sprague BL, Lowry KP, Miglioretti DL, Alsheik N, Bowles EJA, et al. Changes in Mammography Utilization by Women's Characteristics during the First 5 Months of the COVID-19 Pandemic. JNCI: Journal of the National Cancer Institute. 2021.
22. Bleyer A, Welch HG. Effect of Three Decades of Screening Mammography on Breast-Cancer Incidence. New England Journal of Medicine. 2012; 367: 1998-2005.

23. Printz C. Patient protection and affordable care act elimination of mammogram copays has increased mammography rates. Cancer. 2018; 124: 2070.

24. Planning NYC-DoC. Population - Current and Projected Populations. 2021.

25. Surgeons ASoB. Consensus Guideline on Diagnostic and Screening Magnetic Resonance Imaging of the Breast. 2017.

26. Boetes $\mathrm{C}$. Update on screening breast MRI in high-risk women. Magn Reson Imaging Clin N Am. 2010; 18: 241-247.

27. COVID-19: Data. 2020.

28. Seely JM, Alhassan T. Screening for breast cancer in 2018-what should we be doing today? Curr Oncol. 2018; 25: S115-S124

29. Vicini E, Galimberti V, Naninato P, Vento AR, Ribeiro Fontana SK, et al. COVID-19: The European institute of oncology as a \&\#x201c; hub\&\#x201d; centre for breast cancer surgery during the pandemic in Milan (Lombardy region, northern Italy) - A screenshot of the first month. European Journal of Surgical Oncology. 2020; 46: 1180-1181.

30. Veronesi P, Corso G. Impact of COVID-19 pandemic on clinical and surgical breast cancer management. EClinicalMedicine. 2020; 26: 100523.

31. Solomon MD, McNulty EJ, Rana JS, et al. The Covid-19 Pandemic and the Incidence of Acute Myocardial Infarction. New England Journal of Medicine. 2020; 383: 691-693.

32. Garcia S, Albaghdadi MS, Meraj PM, Schmidt C, Garberich R, et al. Reduction in ST-Segment Elevation Cardiac Catheterization Laboratory Activations in the United States during COVID-19 Pandemic. Journal of the American College of Cardiology. 2020; 75: 2871-2872. 